\title{
Bone and joint tuberculosis
}

\author{
Carlos Pigrau-Serrallach • Dolores Rodríguez-Pardo
}

Received: 20 September 2011/Accepted: 17 April 2012/Published online: 19 June 2012

(C) Springer-Verlag 2012

\begin{abstract}
Introduction Bone and joint tuberculosis has increased in the past two decades in relation with AIDS epidemics.

Material and methods A literature review of bone and joint tuberculosis, focusing on Pott's disease.

Results Bone and joint TB comprises a group of serious infectious diseases whose incidence has increased in the past two decades, especially in underdeveloped countries, in part due to the AIDS epidemic. Tuberculous spinal infections should be suspected in patients with an insidious, progressive history of back pain and in individuals from an endemic area, especially when the thoracic vertebrae are affected and a pattern of bone destruction with relative disc preservation and paravertebral and epidural soft tissue masses are observed. Atypical tuberculous osteoarticular manifestations involving the extraspinal skeleton, a prosthetic joint, or the trochanteric area, and nontuberculous mycobacterial infections should be considered in favorable epidemiological contexts. Surgery combined with prolonged specific antituberculous chemotherapy is mainly indicated in patients with neurological manifestations or deformities, and provides satisfactory results in most cases. Conclusions Spinal tuberculosis is still a relative common extra spinal manifestation of spinal tuberculosis that
\end{abstract}

C. Pigrau-Serrallach $(\square)$

Infectious Diseases Division, Hospital Universitari Vall

d'Hebron, Universidad Autónoma, Barcelona REIPI (Spanish

Network for Research in Infectious Disease), $\mathrm{P}^{\circ}$ Vall $\mathrm{d}^{\prime}$ Hebron

119-129, 08035 Barcelona, Spain

e-mail: cpigrau@vhebron.net

D. Rodríguez-Pardo

Adjunto de Enfermedades Infecciosas, Hospital Universitari

Vall d'Hebron, Barcelona REIPI (Spanish Network for Research

in Infectious Disease), Barcelona, Spain requires a high degree of suspicion in order to avoid neurological complications and need of surgery.

Keywords Bone and joint tuberculosis $\cdot$ Spinal tuberculosis - Pott's disease - Tuberculous vertebral osteomyelitis - Mycobacterial bone infections

\section{Introduction}

Bone and joint tuberculosis (TB) is an ancient disease. Evidence of osteoarticular TB has been detected in Egyptian mummies [7, 22, 70], Iron Age remains from Asia $[63,64]$, and skeletons of Europeans living in the Middle Ages [23] by histological or polymerase chain reaction (PCR) study [70, 71].

Mycobacterium tuberculosis is by far the most common cause of mycobacterial osteomyelitis and arthritis worldwide [23]. Nontuberculous mycobacterial (NTM) skeletal infections, although very rare, increased in the 1980s and 1990s in relation to the AIDS epidemic [22]. NTM infections have been associated with a previous injury or puncture wound [34] and with orthopedic surgery, such as hip or knee arthroplasty [56]. Furthermore, in recent years, M. bovis skeletal infections have been reported after intravesical bacillus Calmette Guérin (BCG) therapy [50].

\section{Pathophysiology}

Tuberculous osteomyelitis and arthritis generally arise from reactivation of bacilli lodged in bone during the original mycobacteremia of primary infection. The predilection of the bacillus for the spine and large joints can be explained by the rich vascular supply of the vertebra and 
growth plates of the long bones. Tuberculous arthritis is believed to result from extension of an initial infectious focus in the bone to the joint. Infrequently, tuberculous bacilli travel from the lung to the spine along the Batson paravertebral venous plexus or by lymphatic drainage to the paraaortic lymph nodes [22].

NTM osteoarticular infections usually arise secondary to direct inoculation of mycobacteria following a traumatic injury or during surgical procedures such as joint arthroplasty $[16,34]$. Hematogenous dissemination can occur in immunocompromised patients with NTM bone infections, such as individuals with AIDS or transplant recipients. In some $M$. bovis vertebral infections, the microorganism reaches the vertebral body from the urinary bladder through the Batson venous plexus.

\section{Tuberculous mycobacterial bone infections}

In Europe and the USA, extrapulmonary tuberculosis (EPTB) has increased from $7.6 \%$ of all TB cases in the 1960 s to $20-40 \%$ in recent studies [28, 35, 53]. In children, around $20 \%$ of all mycobacterial infections are EPTB [43]. This increase has been attributed to the epidemic of AIDS, a condition that predisposes to EPTB, and to immigration, EPTB being more common in Asian and African countries [28, 35, 53].

Bone and joint TB currently accounts for 2.2-4.7\% of all TB cases in Europe and the USA and around 10-15\% of EPTB cases (Table 1), whereas in undeveloped countries, particularly Asia, the incidence of EPTB increases to 15-20\% [68]. In Spain, a country with an intermediate incidence of TB, a smaller reduction in the incidence of EPTB than in pulmonary TB was observed for the period of 1991-2008 [21]. Among all EPTB cases documented in that study, $5.8 \%$ were bone and joint infections. In 2004, in the region of Catalunya, $30 \%$ of all TB cases were EPTB, and the skeleton was the fourth most commonly affected site, accounting for $2.1 \%$ of all TB cases [15].

In developed countries, 58-81\% of skeletal TB cases are diagnosed in immigrants (Table 1), and in the USA,
$10 \%$ of EPTB cases occur in HIV-infected patients [53]. In Africa, there is a higher prevalence of HIV infection, and in some regions, up to one-third of adults with osteoarticular infections are HIV positive [5].

There are fewer data in children. In England, Wales, and Northern Ireland, bone and joint TB accounted for $2.4 \%$ of all TB cases in children in the 7-year study period (1999-2006) [29].

Bone and joint TB shows a bimodal age distribution: in natives of developed countries, the disease commonly affects people older than 55 years, whereas in immigrants, it is more common in younger individuals (20-35 years old) $[14,28,35,53]$. In our experience, we have also seen this bimodal distribution. In native Spanish patients, osteoarticular TB in mainly seen in people in their 60s. Younger individuals with the disease are mainly HIVinfected patients or more commonly, immigrants from Asian or African countries.

In patients with skeletal $\mathrm{TB}$, concomitant pulmonary involvement is diagnosed in 6.9-29\% of cases (Table 1) [31, 35, 53].

\section{Tuberculous vertebral osteomyelitis (Pott's disease)}

In our experience, $14.6 \%(19 / 130)$ of spontaneous vertebral osteomyelitis infections (Pott's disease) were caused by $M$. tuberculosis [54]. In other studies, 17-27\% of all spondylodiscitis cases had a mycobacterial etiology [8, 44, 66].

The true incidence of vertebral TB worldwide is unknown, but in developed countries, it has increased in the last decade, also in relation to immigration and the HIV epidemic. Nowadays, 5-92\% of tuberculous vertebral osteomyelitis cases in developed countries occur in immigrants (Table 2). In some studies, none of the patients had concomitant HIV infection, whereas in others [41, 58], a high incidence (25-27\%) of cases occurred in HIV-positive patients. In this subgroup, the clinical presentation and course were similar to the pattern seen in HIV-negative cases, and the development of spinal TB did not seem related to the severity of immunosuppression. Other
Table 1 Epidemiology of bone and joint tuberculosis in developed countries

$E P T B$ extrapulmonary tuberculosis, $T B$ tuberculosis

\begin{tabular}{lrlllll}
\hline Country (year) & $\begin{array}{l}\text { No. of } \\
\text { cases }\end{array}$ & $\begin{array}{l}\text { Incidence } \\
\text { of all TB } \\
(\%)\end{array}$ & $\begin{array}{l}\% \text { of } \\
\text { EPTB } \\
\text { patients }\end{array}$ & $\begin{array}{l}\% \\
\text { immigrants }\end{array}$ & $\begin{array}{l}\text { Concomitant } \\
\text { pulmonary TB } \\
(\%)\end{array}$ & $\begin{array}{l}\text { Spine } \\
\text { involvement } \\
(\%)\end{array}$ \\
\hline Denmark [31] (2000) & 95 & 4 & 15 & $2 / 3$ & - & 50 \\
Netherlands [35] (2004) & 532 & 4.3 & 10.6 & 58 & 29 & 56 \\
England-W [14] (1984) & 198 & 4.7 & - & 60 & 15 & 36 \\
USA [53] (2009) & 5337 & 2.2 & 11.3 & 81 & 6.9 & - \\
Turkey [28] (2011) & 11 & 2.7 & 11 & - & - & - \\
Spain [45] (2007) & 53 & - & - & 15 & 15 & 64 \\
\hline
\end{tabular}


S558

Eur Spine J (2013) 22 (Suppl 4):S556-S566

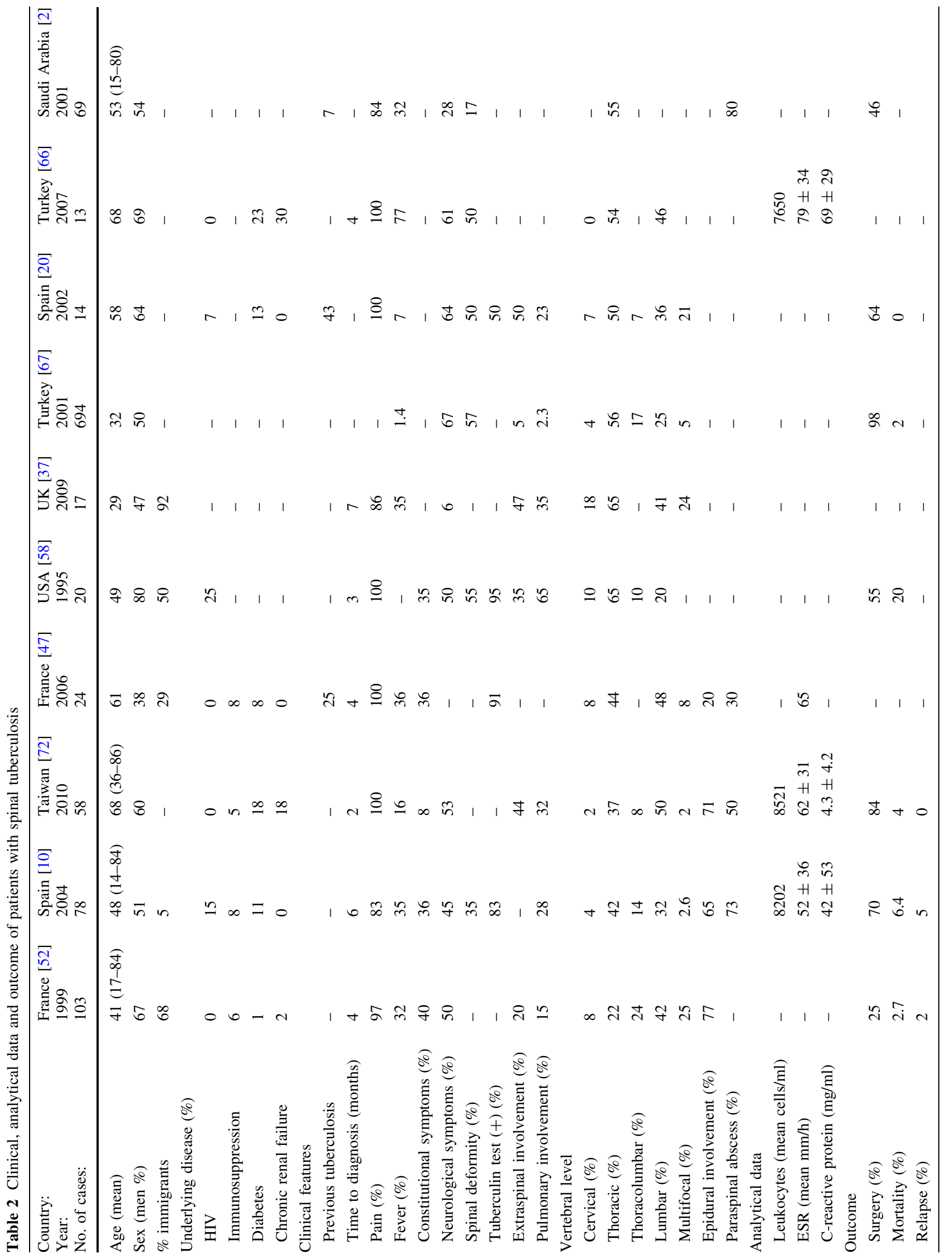

Springer 
predisposing immunosuppressive factors, such as administration of corticosteroids, solid organ neoplasm, and transplantation, predispose to spinal $\mathrm{TB}$ in around 5-7\% of cases. Diabetes mellitus is observed in 1-23\% of patients, and around $2 \%$ have chronic renal failure, although in one small study, the incidence of chronic renal failure was as high as $30 \%$ (Table 2).

\section{Clinical features}

Overall, the mean age of patients with tuberculous vertebral osteomyelitis is 45-60 years (Table 2). Nonetheless, some studies [10] report a bimodal age distribution, with two peaks, one between 20 and 30 years related to immigration and/or HIV infection (in one study, $60 \%$ of cases) and a second between 60 and 70 years. The incidence of concomitant extraspinal involvement varies (5-50\%) and concomitant lung disease is observed in 2.3-65\% (Table 2).

Symptom onset is usually insidious in spinal TB, and disease progression is slow, although an acute onset has been reported [52]. The duration of symptoms at diagnosis ranges from 2 weeks to several years. In earlier studies, the average was at least 12 months, but more recent publications report a symptom duration of 2-7 months (Table 2). Back pain is usually present (83-100\%) (Table 2), but only one-third of patients have fever or constitutional symptoms. These manifestations are more frequent in patients with associated extraspinal $\mathrm{TB}$ and those with disseminated disease.

Tuberculous vertebral osteomyelitis affects the thoracic or thoracolumbar segment in around half of the cases, followed by the lumbar segment, and to a much lesser extent, the cervical segment. Multifocal non-contiguous spinal involvement is reported to be uncommon in some studies (Table 2), but is observed in 16.3-71.4\% of cases when whole-body MRI is performed [32, 36, 55]. Noncontiguous spinal TB is common in areas that have a high prevalence of mycobacterial infection, such as South Africa [55].

\section{Complications}

The frequency of thoracic spine involvement, the insidious course of the infection, and diagnostic delays explain the high incidence of neurological complications, by far the most common complication of spinal TB.

\section{Neurological complications}

Approximately, $50 \%$ of spinal TB patients (Table 2) have neurological manifestations resulting from compression of the spinal cord, and the cauda equina or other nerve roots.
Ten percent to $27 \%$ of patients develop paraplegia or tetraplegia, which are more common in patients with cervical or thoracic spinal TB, with incidences of around $40-50 \%$ [53]. In one study, the incidence of neurological complications was higher in patients with non-contiguous, multilevel spinal TB $(75 \%)$ compared to the remainder of the group studied (58.5\%) [55]. Neurological complications are more common in patients with associated epidural abscess. During active disease, paraplegia can result from mechanical compression on the spinal cord by an abscess, granulation tissue, tubercular debris and caseous tissue, or by mechanical instability produced by pathological subluxation or dislocation [32]. In rare cases, paraplegia is caused by edema of the spinal cord, myelomalacia, or direct involvement of the meninges and cord by tubercular infiltration (tuberculous spinal leptomeningitis), infective thrombosis, or endarteritis of the spinal vessels [32]. Surgical treatment is usually indicated even for minimal neurological deficits. However, in a classic study, Tuli [65] reported neurological recovery in 30-40\% of cases using only rest and antituberculous therapy. Thus, although surgery is usually mandatory, when the neurological deficit is secondary to mechanical compression due to a fluid collection in the extradural space and the spinal cord is relatively preserved (edema without myelomalacia), conservative treatment may be effective. If the neurological deficit persists or worsens, surgical decompression should be performed [32]. In patients with severe deformity, paraplegia can develop months or years after the lesion has healed due to stretching of the spinal cord over an internal anterior bony projection, producing gliosis. In this situation, MRI shows severe cord atrophy or syringohydromyelia, or constrictive scarring of and around the dura [32].

\section{Spinal deformity}

The development of kyphosis is the rule rather than the exception. In patients treated conservatively, the mean increase in deformity is $15^{\circ}$, and in $3-5 \%$, the final deformity is $>60^{\circ}$ [32]. In children, kyphosis continues to increase even after the lesion has healed [32, 56]. In $40 \%$ of these patients [57], the deformity progresses during the quiescent phase until completion of growth, whereas $43 \%$ improve spontaneously. In children younger than 7 years of age with three or more affected vertebral bodies in the thoracic spine and two or more radiological at-risk signs, kyphosis is likely to progress with growth; hence surgery should be considered [32, 57]. Progression of kyphosis can also occur after surgery and is worse when anterior resection and fusion alone are performed. It is less severe when surgery includes both anterior and posterior fusion. 


\section{Other complications}

In large series (Table 2), paraspinal involvement is observed in 50-80\% of cases and epidural masses are seen in around $70 \%$. These imaging findings are detected more often when MRI is performed because of its high sensitivity. A concomitant psoas abscess is a relatively frequent finding, but its true incidence is unknown; in one study, the incidence of this complication was as high as $24.4 \%$ [9]. In exceptional cases of cervical spine TB with a large retropharyngeal abscess, hoarseness and problems with swallowing may occur. A rare complication of Pott's disease is tubercular pseudoaneurysm of the aorta, secondary to extension of an adjacent tubercular vertebral lesion or due to tubercular arteritis [33].

\section{Laboratory data}

Hematological and clinical data contribute little to the diagnosis, and leukocyte count is usually normal. The erythrocyte sedimentation rate (ESR) and C-reactive protein concentration are often raised, but levels are lower than those seen in pyogenic vertebral infections. The tuberculin skin test is usually positive, although it is nondiagnostic in endemic regions and may be negative in immunodeficient patients (Table 2).

\section{Diagnosis and differential diagnosis}

Spinal TB can be suspected both clinically and radiologically. A pattern of bone destruction showing a low signal on T1-weighted MRI and a bright signal on T2-weighted images in affected vertebral bodies with relative preservation of the disc and heterogeneous enhancement may differentiate spondylitic TB from pyogenic discitis, which usually shows peridiscal bone destruction and homogeneous enhancement.

The definitive diagnosis of spinal TB (discussed in detail in another chapter) is usually established by CT-guided needle aspiration cytology-biopsy and culture on Löwenstein medium (sensitivity of 50-75\%), or by histological examination, which is highly suggestive of spinal TB when caseating granulomas are observed and diagnostic when acid-fast bacilli are found (sensitivity around $70 \%$ ). Culture and histological study of a bone specimen obtained by surgery has a slightly higher diagnostic yield [10].

Tuberculous spondylitis (TS) should be differentiated from primary or metastatic neoplastic disease, which usually affects the bone (vertebral body) and spares the intervertebral disc (except in primary vertebral myeloma, some cases of lymphoma, and rare cases of solid organ tumors such as thyroid neoplasms), pyogenic spondylitis (PS), and in endemic areas, brucellar spondylitis (BS). Although blood cultures are often negative in BS [9] and agglutination titers may be low (especially in patients with prolonged disease), the anti-Brucella Coombs test shows high titers and easily excludes the diagnosis of BS. The differential diagnosis with PS is more difficult since blood cultures are negative in 30-40 \% of PS cases [54, 69] and CT-guided or open biopsy cultures have a diagnostic yield of $77 \%$ [49]. In TS, the diagnostic yield of CT-guided biopsy and mycobacterial culture is around $75 \%[11,44,49,52]$. Histopathological analysis may also be useful. The presence of leukocytes distinguishes infection from contamination, and granulomas suggest TB or brucellosis [70], although granulomas are absent in one-third of TS cases [8].

Given the fact that in PS and TS an etiological diagnosis is not always obtained even when invasive techniques are used, some authors have investigated clinical, analytical and radiological data that can help to distinguish between the two conditions [9, 66]. Colmenero et al. [9] have reported that diabetes mellitus, drug abuse, underlying immunosuppression or chronic debilitating diseases, previous localized infection or bacteremia, leucocytosis, and a high ESR are significantly associated with PS. In contrast, a prolonged clinical course, thoracic spine involvement, absence of fever, presence of spinal deformity or neurological deficits, and paravertebral or epidural masses are more frequent in TS. Calcifications in soft tissue masses and vertebral collapse with relative preservation of the intervertebral disc have been considered characteristic of TS [10]. In addition, Turunc et al. [66] reported an association of chronic renal failure, psoas abscess, and involvement of the posterior elements with TS.

Molecular techniques such as multiplex real-time PCR may be useful for rapid diagnosis of TS and BS [8] and can distinguish typical from atypical mycobacteria. Nonetheless, these techniques are not indicative of disease activity because they cannot differentiate between living and dead microorganisms.

\section{Treatment}

In spinal TB with no neurological deficit, conservative treatment with a combination of antituberculous drugs yields similar long-term results, with no late relapse or lateonset paraplegia, when compared to surgery with anterior debridement and fusion or anterior debridement alone [32]. Surgical and medical therapies are specified in other chapters.

\section{Outcome}

The reported mortality of spinal TB is usually low (0-6\%), except in one study performed in the USA in which a mortality rate of $20 \%$ was described [58]. However, this 
study included a large number of HIV-positive patients with concomitant pulmonary TB and disseminated disease. With adequate antituberculous chemotherapy and surgery when required, relapses are uncommon (0-5 \%). Surgery, which is mainly indicated for neurological complications or spinal deformity-instability, is needed in more than $50 \%$ of cases (Table 2). In patients with a delayed diagnosis, surgical requirements may be as high as $98 \%$. This was the case in a multicenter study performed in Turkey in which patients with Pott's disease unfortunately had large abscesses, a high incidence of neurological complications and spinal deformity, and multisegmental involvement when admitted to hospital [67].

\section{Atypical spinal tuberculosis}

The typical MRI pattern of spinal TB shows vertebral bone destruction with relative preservation of the disc, whereas in pyogenic infection the disc is usually markedly affected, there are septate prevertebral and paravertebral or intraosseous abscesses with a subligamentous extension, and breaching of the epidural space is seen [1, 13, 63]. Atypical spinal TB is uncommon and includes cases of compressive myelopathy with no visible or palpable spinal deformity and no radiological signs of a typical tuberculous vertebral lesion. This form of spinal TB may mimic lowgrade pyogenic infection, brucellar, hydatidic or sicklecell spondylitis, or lymphomatous or malignant vertebral disease, and be difficult to diagnose and treat at early stages; hence, neurological complications are common [32]. Atypical lesions can also present as intraspinal tubercular granulomas, a sole lesion of the posterior elements, compressive myelopathy in single vertebral disease, a collapsed vertebral body, or as a sclerotic vertebra with bridging of the intervertebral body [32].

Granulomatous lesions of the epidural, intradural, or intramedullary spaces can cause spinal tumor syndrome with compressive myelopathy; no obvious vertebral bone alterations are seen on radiological study. Patients with extradural or subdural granuloma usually recover well following surgery for the histological diagnosis and laminectomy to decompress the spinal cord [32]. The usual clinical presentation of intramedullary granulomatous TB is a painless compressive myelopathy that should be differentiated from solid spinal cord tumors and cysticercosis. Surgical decompression and myelotomy are indicated to decompress the spinal cord and establish the diagnosis [32].

In endemic areas, 2-10\% of all patients with vertebral TB have a single lesion in the posterior elements [3, 32]. The lesion can affect the spinous process, laminae, apophyseal joints, transverse processes, or the pedicles, the most common location. The incidence of paraplegia is high in this atypical form, and primary or metastatic tumoral disease is initially suspected. Fine-needle aspiration cytology and biopsy can be useful for the diagnosis. Antituberculous chemotherapy suffices in patients with no neurological deficit, and laminectomy is indicated in those with neurological involvement.

Tuberculous infection can start in the center of the vertebral body, and present with pain and concentric vertebral body collapse with preservation of the disc. The differential diagnosis should be established with other conditions, such as osteoporosis, traumatic injury, and neoplastic disease. The diagnosis is usually attained by CT-guided biopsy.

\section{Peripheral osteoarticular tuberculosis}

Peripheral osteoarticular tuberculosis is the term used for extraspinal skeletal TB that affects joints or bones. Long weight-bearing bones are usually affected, knee or hip arthritis is relatively common, and femoral disease (including trochanteric tuberculous bursitis) is not rare (Table 3). The ribs and sacroiliac and sternoclavicular joints are sometimes affected. TB is one of the most common causes of rib osteomyelitis. Extraspinal tuberculous osteomyelitis presents as a cold abscess, with swelling and only mild erythema and pain, and may be misdiagnosed as a tumor.

Table 3 Localization of bone and joint tuberculosis

\begin{tabular}{lcclc}
\hline References: & $\begin{array}{l}\text { Mateo } \\
{[45]}\end{array}$ & $\begin{array}{l}\text { Enache } \\
{[18]}\end{array}$ & $\begin{array}{l}\text { Houshian } \\
{[31]}\end{array}$ & $\begin{array}{l}\text { Davies } \\
{[14]}\end{array}$ \\
\hline Localization (\%) & & & & \\
Spine & 64 & $0^{\mathrm{a}}$ & 49 & 36 \\
Knee & 8 & 11 & 7 & 11 \\
Ankle & 8 & 8 & 0 & 8 \\
Elbow-arm & 2 & 11 & 5 & 5 \\
Wrist-hand & 6 & 26 & 4 & 11 \\
Hip & 0 & 10 & 6 & 9 \\
Rib & 2 & 26 & 2 & 1 \\
Sternoclavicular & 6 & 0 & 3 & 2 \\
Sacroiliac & 9 & 0 & 0 & 4 \\
Foot & 0 & 0 & 5 & 3 \\
Tibia & 0 & 0 & 2 & 2 \\
Trochanter- & 6 & 10 & 5 & 2 \\
$\quad$ femur & & & & 5 \\
Others & 0 & 5 & 3 &
\end{tabular}

a The low incidence of spinal tuberculosis is explained by the fact that vertebral osteomyelitis is usually treated in specialized referral centers in the country of the study 
Articular TB is a slowly progressive chronic disease that presents as monoarthritis, usually of the hip or knee, in $90 \%$ of cases [25] (Table 3). Previous traumatic injury may be the precipitating event. The earliest manifestation is pain, which may precede signs of inflammation for weeks or months. Fever and systemic symptoms are usually absent.

Radiography may initially show soft tissue swelling, but later osteopenia, periosteal thickening, and periarticular bone destruction is observed. Cold abscesses and fistulae develop in late cases. Chest radiography shows pulmonary disease in one-third to one half of cases, but active pulmonary TB is infrequent [25]. The early stages are often misdiagnosed, and the joint disease is attributed to traumatism, degenerative disease, gout or pseudogout, rheumatic disease such as rheumatoid arthritis, or pigmented villonodular synovitis. The diagnosis requires a high index of suspicion and is usually established through arthrocentesis and mycobacterial culture; however, synovial biopsy is often needed. In early cases, prolonged antituberculous therapy results in complete resolution. Surgery is necessary in advanced cases, which may require arthrodesis or arthroplasty of the joint.

TB can also affect the tendon sheaths and is one of the causes of tenosynovitis of the hand and carpal tunnel syndrome. In a recent study, 2/27 patients with tuberculous arthritis had concomitant tenosynovitis [42]. The diagnosis is usually delayed because $M$. tuberculosis is uncommon etiology of these conditions.

\section{Special osteoarticular tuberculous infections}

\section{Prosthetic joint infection}

Prosthetic joint infection (PJI) due to M. tuberculosis is rare and has been reported as case studies [4, 62]. The typical case is a misdiagnosed patient who presents with knee or hip osteoarthritis, is treated with joint arthroplasty, and later develops culture-negative chronic PJI, sometimes years after the procedure [62]. The diagnosis is often difficult and should be suspected in culture-negative PJI with histological features of granulomatous lesions with macrophages and multinucleate cells with or without caseum. The diagnosis is confirmed by isolation of the microorganism on Löwenstein culture or by molecular techniques (PCR). In some cases, administration of immunosuppressive therapy is the precipitating event, as we observed in one patient with rheumatoid arthritis and a hip prosthesis who developed PJI due to M. tuberculosis and Salmonella enteritidis after administration of anti-tumor necrosis factor (infliximab).

Resection arthroplasty or arthrodesis has been used to treat this type of PJI, but when there is no loosening of the prosthesis, the patient may cure with debridement, exchange of plastic components while retaining the prosthesis, and prolonged antituberculous therapy (9-12 months). In our patient, prosthetic debridement was performed, the polyethylene components were removed, and antituberculous chemotherapy with isoniazid, rifampin, and levofloxacin was administered for 12 months. She has been followed-up for more than 8 years and there has been no relapse.

\section{Sternal tuberculosis}

Tuberculosis of the sternum is rarely reported and accounts for $1.5 \%$ of bone and joint TB cases [24, 60]. Primary sternal TB is relatively more common than TB secondary to cardiopulmonary surgery [24]. The diagnosis should be suspected in recurrent culture-negative nonhealing sternal wounds, and confirmed by histology and specific culture. The duration of therapy has not been well established in these patients, and surgery is justified whenever there is a doubt about the diagnosis, in refractory cases, to remove a large sequestrum, or when there are large abscesses.

\section{Tuberculous sacroiliitis}

The sacroiliac joint is affected in $4-9.5 \%$ of patients with musculoskeletal TB [51, 59]. Misdiagnosis is common (92\% in one study) and has been attributed to the inaccessibility of the sacroiliac joint. Tuberculous sacroiliitis should be differentiated from pyogenic sacroiliac bone infections (usually acute), and from chronic diseases (e.g, osteoarthritis), inflammatory diseases (e.g., rheumatoid arthritis), ankylosing spondylitis and Reiter's disease, gout and pseudogout, tumor-like conditions (e.g., pigmented villonodular synovitis), and in endemic areas, from brucellar sacroiliitis. Currently, ant-tuberculous therapy is the treatment of choice. Surgery (arthrodesis) is used in patients with large periarticular abscesses and those with persistent pain.

\section{Tuberculous trochanteric bursitis}

Primary TB of the trochanteric area, a relatively common manifestation of the disease in the pre-antituberculosis drug era, is now a rare condition accounting for less than $2 \%$ of musculoskeletal TB cases [19]. In our hospital, $0.26 \%$ of TB patients were diagnosed with trochanteric bursitis, an incidence that may be higher than expected because our hospital is a regional referral center [12]. The pathogenesis of tuberculous trochanteric bursitis (TTB) has not been well defined, and it is uncertain whether the bone or the bursa is first affected by hematogenous infection. Recent trauma, immunosuppression, or local corticosteroid infiltrations may contribute to spread the disease. Although this musculoskeletal manifestation has been observed in 
patients of all ages, the mean age at presentation is reported at 57 years [12]. Mild pain, swelling, and stiffness in the lateral aspect of the thigh with no functional limitations are often present for months before the diagnosis is established. Fever or general symptoms are usually absent. This dearth of symptoms, together with a low clinical suspicion, accounts for the usual diagnostic delay of several months (10.5 months in our review) [12]. The diagnosis is commonly made in advanced phases, when a cold abscess or draining fistula that does not respond to conventional antibiotic therapy alerts the clinician.

TTB has a marked tendency to relapse, and antituberculous therapy alone has only been successful at early stages of the disease and in some cases. Surgery should be considered for all patients. Thorough excision of the bursa and all necrotic tissue and fistulous tracks is mandatory for cure. With regard to bone, extensive trochanteric excision has higher resolution rates than bone curettage [12, 19]. The precise duration of antituberculous therapy after extensive surgical excision has not been established; patients are usually treated for 6-12 months.

\section{Multifocal osteoarticular tuberculosis}

Multifocal bone TB is considered rare, accounting for $7-11 \%$ of osteoarticular TB cases [17, 39, 45, 48], but may be higher in undeveloped Asian and African countries, reaching an incidence of $15 \%$. Four to 6 bones or joints are usually affected, but as many as 19 different locations were described in a recent case report [30]. In children, the flat bones of the hand and foot are most commonly affected [17], but spinal involvement has also been reported [45]. Whole-body scintigraphy may be useful to detect occult lesions. The duration of antituberculous therapy in these extensive bone infections is unknown, but most patients are treated for longer than 24 months.

\section{Nontuberculous mycobacterial infections}

Approximately 60 of the more than 125 nontuberculous mycobacterial species can cause disease in humans [34].
NTM have been classified into four groups (Table 4). Previously, identification of NTM species was a tedious process involving biochemical tests that required weeks of culturing. Nowadays, the most relevant species can be identified by molecular techniques.

The prevalence of NTM infections is unknown. M. avium-intracellulare (MAI) is the most commonly isolated NTM species, mainly in patients with HIV infection/AIDS [16, 34]; M. fortuitum and M. kansasii are also relatively frequent.

The spectrum of clinical infections by NTM includes chronic pulmonary infection, superficial lymphadenitis (MAI, M. scrofulaceum), and disseminated disease (MAI), commonly found in advanced HIV patients with a CD4 cell count of less than 50 per $\mu \mathrm{L}$. Other NTM, such as M. abscessus, M. chelonae, M. xenopi, and M. kansasii, can cause disseminated disease in HIV-infected and -noninfected patients with severe immunosuppression, such as organ transplant recipients and patients receiving long-term steroid treatment.

NTM soft tissue infections are common, but skeletal disease is rare. Soft tissue infections are usually the result of direct inoculation occurring during penetrating trauma, open surgery (such as mediastinitis and sternal wound infections after cardiothoracic surgery), after injection of steroids or local anesthetics, or following cosmetic surgery, such as abdominoplasty and liposuction [16, 34]. Rapidly growing mycobacteria (e.g, M. abscessus, M. chelonae, M. fortuitum) are the most commonly isolated agents.

The clinical course is usually indolent, with painful red to violaceous nodules that can drain serosanguineous material, ulcerate, or spread to deeper tissues and form fistulous tracts. The histological study shows abundant neutrophils and caseum, although non-caseating granulomas may be also observed. The acid-fast bacilli test is usually negative. Some species are associated with specific clinical syndromes. This is the case of M. marinum, which causes a chronic granulomatous infection known as swimming pool granuloma or fish tank granuloma and is observed in individuals exposed to a marine environment or marine products (fish, crustaceans, fish tanks). The

Table 4 Runyon classification of nontuberculous mycobacteria

\begin{tabular}{|c|c|c|c|c|}
\hline Class & Description & Growth & Pigment production & Main species \\
\hline I & Photochromogens & Slow & $\begin{array}{l}\text { Yellow-orange pigment when } \\
\text { exposed to light }\end{array}$ & M. kansasii, M. marinum \\
\hline II & Scotochromogens & Slow & $\begin{array}{l}\text { Yellow-orange pigment with } \\
\text { or without light }\end{array}$ & M. scrofulaceum, M.szulgai, M. gordonae \\
\hline III & Nonchromogens & Slow & None & M. avium-intracellulare, $M$. xenopi, $M$. terrae \\
\hline IV & Rapid growers $(<7$ days $)$ & Rapid & None & M. fortuitum, M. chelonae M. abscessus \\
\hline
\end{tabular}


lesions begin as papules that ulcerate and scar. The infection can spread and lead to a nodular lymphangitis that is similar to sporotrichosis lymphangitis. In the tropics and Australia, M. ulcerans causes the so-called Buruli ulcer, a chronic necrotic skin lesion that ulcerates.

Skeletal infections due to NTM are rare and have been observed in cluster outbreaks in cardiothoracic surgery (sternal osteomyelitis due to M. fortuitum or M. abscessus) or in isolated cases of M. xenopi arthritis [6] after joint arthroplasty. Our experience includes one non-immunosuppressed patient with a knee prosthesis, who developed an early postoperative knee infection due to M. xenopi. In immunosuppressed patients with disseminated disease, the bone may be affected, as we observed in an HIV-infected woman with disseminated $M$. avium-intracellulare infection, who developed chronic tibial NTM osteomyelitis due to $M$. avium-intracellulare with a fistulous sinus. She was cured with bone resection and lengthy (18 months) specific antituberculous therapy. We also diagnosed an NTM bone infection in a lung transplant recipient who had disseminated mycobacteremia due to M. abscessus and developed relapsing septic knee arthritis due to the same microorganism. The patient died of disseminated mycobacterial infection despite repeated surgical knee drainage and specific antituberculous therapy.

For osteoarticular infections due to NTM, surgical excision of the infected tissue and/or prosthetic joint removal should be performed [27]. A minimum of 6 months of specific antimycobacterial chemotherapy is recommended, and the regimen can be extended to 12 or more months in patients with disseminated disease.

\section{Mycobacterium bovis BCG osteoarticular infections}

Intravesical instillation of bacillus Calmette Guérin (BCG), first introduced in 1976 to treat superficial bladder carcinoma, can be associated with significant adverse events, such as immunologically mediated fever $(2.9 \%)$, BCG sepsis $(0.4 \%)$, granulomatous prostatitis or epididymoorchitis $(1 \%)$, granulomatous hepatitis or pneumonitis $(<1 \%)$, immunocomplex glomerulonephritis and allergic reactions with skin rash $(0.3 \%)$, and arthritis/ arthralgias $(0.5 \%)$ [38, 40]. M. bovis BCG intravesical instillations have been anecdotally associated with skeletal infections, particularly spondylitis [26, 46, 50], which may present as late as 12 years after the procedure, and with hip or knee arthroplasty [61]. The diagnosis is established by culture and recently, by PCR-based genomic analysis. Because M. bovis is resistant to pyrazinamide, the usual antimycobacterial regimen consists of isoniazid, rifampin, and ethambutol administration for as long as 12 months $[46,61]$.
In conclusion, bone and joint TB comprises a group of serious infectious diseases whose incidence has increased in the last two decades, especially in underdeveloped countries, in part due to the AIDS epidemic. Tuberculous spinal infections should be suspected in patients with an insidious, progressive history of back pain and in individuals from an endemic area, especially when the thoracic vertebrae are affected and a pattern of bone destruction with relative disc preservation and paravertebral and epidural soft tissue masses are observed. Atypical tuberculous osteoarticular manifestations involving the extraspinal skeleton, a prosthetic joint, or the trochanteric area, and nontuberculous mycobacterial infections should be considered in favorable epidemiological contexts. Surgery combined with prolonged specific antituberculous chemotherapy is mainly indicated in patients with neurological manifestations or deformities, and provides satisfactory results in most cases.

Acknowledgments English language editing was done by Celine Cavallo.

Conflict of interest Both the authors have no potential conflict of interest.

\section{References}

1. Andronikou S, Jadwat S, Douis H (2002) Patterns of disease on MRI in 53 children with tuberculous spondylitis and the role of gadolinium. Pediatr Radiol 32:798-805

2. Alothmqan A, ZiadM Awada A, Al-mahmood S, Al-shadoon S, Rahman M, Khan M (2001) Tuberculous spondylitis: analysis of 69 cases from Arabia Saudi. Spine 26:E565-E570

3. Babhulkar SS, Tayade WB, Babhulkar SK (1984) Atypical spinal tuberculosis. J Bone Joint Surg (Br) 66-B:239-242

4. Berbari EF, Hanssen AD, Duffy MC, Steckelberg JM, Osmon DR (1998) Prosthetic joint infection due to Mycobacterium tuberculosis: a case report and review of the literature. Am J Orthop 27:219-227

5. Biviji A, Paiement G, Steinbach L (2002) Musculoskeletal manifestations of human immunodeficiency virus infection. J Am Acad Orthop Surg 10:312-320

6. Brown-Elliot B (2010) Infections due to nontuberculous mycobacteria other than Mycobacterium avium intracellulare. In: En Mandell L, Bennet JE, Dolin R (eds) Principles and practice of infectious diseases, 7th edn. Churching Livingstone-Elsevier, Philadelphia, pp 3191-3198

7. Chastel C (2004) When the Egyptian mummies are speaking about the infections that have made them ill. Hist Sci Med 38:147-155

8. Colmenero JD, Morata P, Ruiz-Mesa JD, Bautista D, Bermúdez P, Bravo MJ, Queipo-Ortuño MI (2010) Multiplex real-time polymerase chain reaction. A practical approach for rapid diagnosis of tuberculous and brucellar vertebral osteomyelitis. Spine 35:E1392-E1396

9. Colmenero JD, Jiménez-Mejías ME, Sánchez-Lora FJ, PalominoNicás J, Martos F, GarcíadelasHeras J, Pachón J (1997) Pyogenic, tuberculous, and brucellar vertebral osteomyelitis: a descriptive and comparative study of 219 cases. Ann Rheum Dis 56:709-715 
10. Colmenero JD, Jiménez-Mejías ME, Reguera JM, PalominoNicás J, Ruiz-Mesa JD, Márquez-Rivas J, Lozano A, Pachón J (2004) Tuberculous vertebral osteomyelitis in the new millennium: still a diagnostic and therapeutic challenge. Eur J Clin Microbiol Infect Dis 23:477-483

11. Cottle L, Riordan $T$ (2008) Infectious spondylodiscitis. J Infect 56:401-412

12. Crespo M, Pigrau C, Flores X, Almirante B, Falcó F, Vidal A, Pahissa A (2004) Tuberculous trochanteric bursitis: report of 5 cases and literature review. Scand J Infect Dis 36:552-558

13. Danchaivitr N, Temran S, Thepmongkhol K, Chiewvit P (2007) Diagnostic accuracy of MRI in tuberculous spondylitis. J Med Assoc Thail 90:1581-1589

14. Davies P, Humpries MJ, Byfield SP, Nunn AJ, Darbyshire JH, Citron KM, Fox W (1984) Bone and Joint tuberculosis. A survey of notifications in England and Wales. J Bone Joint Surg B 66:326-330

15. Departament de Salut (2004) Situació epidemiológica I tendencia de la endemia tuberculosa a Catalunya. Infrome annual 2004. Barcelona: department de Salut. Generalitat de Catalunya

16. De Groote MA, Huit G (2006) Infections due to rapidly growing mycobacteria. Clin Infect Dis 42:1756-1763

17. Dhammi I, Jain A, Singh S, Aggarwal A, Kumar S (2003) Multifocal skeletal tuberculosis in children: a retrospective study of 18 cases. Scand J Infect Dis 35:797-799

18. Enache S, Plesea I, Anusca D, Zaharia B, Pop O (2005) Osteoarticular tuberculosis: a ten years case review. Rom J Morphol Embryol 46:67-72

19. Franceschi P, Chapuis J, Curvale G, Roux H, Aquaviva P, Groulier P (1991) Trochantérites bacillaires: a propôs of 30 cases. Ver Rhum 58:433-439

20. García-Lechuz J, Julve R, Ruiz-Serrano J, Muñoz P (2002) Espondilitis tuberculosa o enfermedad de Pott: experiencia en un hospital general. Enf Infecc Microbiol Clin 20:5-9

21. García-Rodríguez JF, Alvarez-Díez H, Lorenzo-García M, Mariño-Callejo L, Fernández-Rial A, Sesma-Sánchez P (2011) Extrapulmonary tuberculosis: epidemiology and risk factors. Enf Infecc Microbiol Clin 29:502-509

22. Gardam M, Lim S (2005) Mycobacterial osteomyelitis and arthritis. Infect Dis Clin N Am 19:819-830

23. Good RC, Snide DE (1982) Isolation of nontuberculous mycobacteria in the United States 1980. J Infect Dis 146:829-833

24. Gopal K, Raj A, Rajesh MR, Prabhu SK, Geothe J (2007) Sternal tuberculosis after sternotomy for coronary artery bypass surgery: a case report and review of the literature. $\mathrm{J}$ Thorac Cardiovasc Surg 133:1365-1366

25. Golden M, Vikram H (2005) Extrapulmonary tuberculosis: an overview. Am Fam Physician 72:1761-1768

26. Gonzalez O, Musher D, Brar I, Furgeson S, Boktour M, Septimus EJ, Hamill RJ, Graviss EA (2003) Spectrum of bacilli CalmetteGuérin (BCG) infection after intravesical BCG immunotherapy. Clin Infect Dis 36:140-148

27. Griffith DE, Aksamit T, Brown-Elliot BA, Catanzaro A, Daley C, Gordin F, Holland SM, Horsburgh R, Huitt G, Lademarco MF, Iseman M, Olivier K, Ruoss S, von Reyn CF, Wallace RJ Jr, Winthrop K (2007) An official ATS/DSA statement: diagnosis, treatment and prevention of nontuberculous mycobacterial diseases. Am J Respir Crit Care Med 175:367-416

28. Gunal S, Yang Z, Agarwal M, Koroglu M, Arici ZK, Durmaz R (2011) Demographic and microbial characteristics of extrapulmonary tuberculosis cases diagnosed in Malatya, Turkey, 2001-2007. BMC Public Health 11:154-161

29. Holland TS, Sangster MJ, Paton RW, Ormerod LP (2010) Bone and join tuberculosis in children in the Blackburn area since 2006: a case series. J Child Orthop 4:67-71
30. Hoing L, Wu J, Ding J, Wang X, Zheng M, Fu R, Li WB, Peng WX, He WF (2010) Multifocal skeletal tuberculosis: experience in diagnosis and treatment. Med Mal Infect 40:6-11

31. Houshian S, Poulsen S, Riegels-Nielsen PR (2000) Bone and joint tuberculosis in Denmark. Increase due to immigration. Acta Orthop Scand 71:312-315

32. Jain AK (2010) Tuberculosis of the spine. J Bone Joint Surg (Br) 92-B:905-913

33. Jain AK, Chauhan RS, Dhammi IK, Maheshawari AV, Ray R (2007) Tubercular pseudoaneurysm of aorta: a rare association with vertebral tuberculosis. Spine J 7:249-253

34. Jarzembowski JA, Young MB (2008) Nontuberculous mycobacterial infections. Arch Pathol 132:1330-1334

35. Jutte PC, Louenhout-Royackers JH, Borgdorf MW, Horn JR (2004) Increase of bone and joint tuberculosis in the Netherlands. J Bone Joint Surg 86:901-904

36. Kaila R, Am Malhi, Mahmood B, Saifuddin A (2009) The incidence of multiple level non-contiguous vertebral tuberculosis detected using whole spine MRI. Eur Spine J 18:1096-1101

37. Kenyon P (2009) Tuberculous vertebral osteomyelitis. Findings of a 10-year review of experience in a UK centre. J Infect 59:372-373

38. Koya MP, Simon MA, Soloway MS (2006) Complications of intravesical therapy for urothelial cancer of the bladder. J Urol 175:2004-2010

39. Kumar K, Saxema MB (1988) Multiple osteoarticular tuberculosis. Int Orthop 12:135-138

40. Lamm DL, Meijden V, Morales APM, Brosman SA, Catalona WJ, Herr HW, Soloway MS, Steg A, Debruyne FM (1992) Incidence and treatment of complications of bacillus CalmetteGuerin intravesical therapy in superficial bladder cancer. J Urol 147:596-661

41. Leibert E, Schluger NW, Bonk S, Rom WN (1996) Spinal tuberculosis in patients with immunodeficiency virus infection. Clinical presentation, therapy and outcome. Tuber Lung Dis 77:329-334

42. Lertsrisatit P, Nantiruj K, Totemchokyakam K, Janwityanujit S (2007) Extraspinal tuberculous arthritis in HIV era. Clin Rheumatol 26:319-321

43. Le Roux P, Quinque K, Bonnel AS, Luyer B (2005) Les atteintes extrapulmonaries de la tuberculose de l'enfant. Arch Pediatr 12:S122-S126

44. Luzzati R, Giacomazzi D, Danzi MC, Tacconi L, Concia E, Vento S (2009) Diagnosis, management and outcome of clinically-suspected spinal infection. J Infect 58:259-265

45. Mateo L, Ruiz J, Olive A, Manterola J, Pérez R, Tena X, Prats M (2007) Tuberculosis osteoarticular. Med Clin (Barc) 129:506-509

46. Mavrogenis AF, Sakellariou V, Tsiodras S, Papagelopulos PJ (2009) Late Mycobacterium bovis spondylitis after intravesical BCG therapy. Joint Bone Spine 76:296-300

47. Mulleman D, Mammou S, Griffoul I, Avimadje A, Goupille P, Vallat JP (2006) Characteristics of patients with spinal tuberculosis in a French teaching hospital. Joint Bone Spine 73:424-427

48. Muradali D, Gold WL, Vellend H, Becker E (1993) Multifocal osteoarticular tuberculosis: report of four cases and review of management. Clin Infect Dis 17:204-205

49. Mylona E, Samarkos M, Kakalou E, Fanourgiakis P, Skoutelis A (2009) Pyogenic vertebral osteomyelitis: a systematic review of clinical characteristics. Sem Arthr Rheum 39:10-17

50. Nikaido T, Ishibashi K, Otani K, Yabuki S, Konno S, Mori S, Ohashi K, Ishida T, Nakano M, Yamaguchi O, Suzutani T, Kikuchi S (2007) Mycobacterium bovis BCG vertebral osteomyelitis after intravesical BCG therapy, diagnosed by PCR-based genomic deletion analysis. J Clin Microbiol 45:4085-4087

51. Papagelopoulos P, Papadopoulos E, Mavrogenis A, Themistocleous G, Korres D, Soucacos P (2005) Tuberculous sacroiliitis. A case report and review of the literature. Eur Spine J 14:683-688 
52. Pertuiset E, Beaudreuil J, Liote F, Horusitsky A, Kemiche F, Richette P, Clerc-Weyl D, Cerf I, Dorfmann H, Glowinski J, Crouzet J, Ziza JM, Bardin T, Meyer O, Dryll A, Kahn MF, Kuntz D (1999) Spinal tuberculosis in adults. A study of 103 cases in developed country, 1980-1994. Medicine (Baltimore) 78:309-320

53. Peto HM, Pratt RH, Harrington TA, Lobue PA, Armstrong LR (2009) Epidemiology of extrapulmonary tuberculosis in the United States, 1993-96. Clin Infect Dis 49:1350-1357

54. Pigrau C, Almirante B, Flores X, Falcó V, Rodríguez D, Gasser I, Villanueva C, Pahissa A (2005) Spontaneous pyogenic vertebral osteomyelitis and endocarditis: incidence, risk factors and outcome. Am J Med 118:1287-1292

55. Polley P, Dunn R (2007) Noncontiguous vertebral tuberculosis detected using whole spine MRI. J Spinal Disord Tech 20: 78-81

56. Pring M, Eckhoff DG (1996) Mycobacterium chelonae infection following a total knee arthroplasty. J Arthroplast 11:115-116

57. Rajasekaran S (2001) The natural history of post-tubercular kyphosis in children: radiological signs which predict late increase in deformity. J Bone Joint Surgery (Br) 83-B:954-962

58. Rezai A, Lee M, Cooper P, Tomas E, Koslow M (1995) Modern management of spinal tuberculosis. Neurosurgery 36:87-97

59. Richter R, Nubling W, Kohler G, Iljinski A (1983) Tuberculosis of the iliosacral joints. Treatment, results, prognosis, and differential diagnosis. Z Orthop Ihre Grenzgeb 121:564-570

60. Rubinstein EM, Lehman T (1996) Sternal osteomyelitis due to Mycobacterium tuberculosis following coronary artery by pass surgery. Clin Infect Dis 23:202-203

61. Segal A, Krausse ES (2007) Infected total hip arthroplasty after intravesical bacillus Calmette-Guerin therapy. J Arthroplasty 22:759-762
62. Shanbhag V, Kotwal R, Gaitonde A, Singhal K (2009) Total hip replacement infected with Mycobacterium tuberculosis. A case report with review of the literature. Acta Orthop Belg 75:119-121

63. Sharief HS, Clarc DC, Ashbed MY, Haddad MC, alDeeb SM, Yaqub B, alMoutaery KR (1990) Granulomatous spinal infections. MR imaging. Radiology 177:101-107

64. Tayles N, Buckley HR (2004) Leprosy and tuberculosis in Iron Age Southeast Asia. Am J Phys Anthropol 125:239-256

65. Tuli SM (1969) Treatment of neurological complications in tuberculosis of the spine. J Bone Joint Surg (Am) 51-A:680-692

66. Turunc T, Demiroglu Y, Uncu H, Colakoglu S, Arsian H (2007) A comparative analysis of tuberculous, brucellar and pyogenic spontaneous spondylodiscitis patients. J Infect 55:158-163

67. Turgut M (2001) Spinal tuberculosis (Pott's disease): its clinical presentation, surgical management, and outcome. A survey study on 694 patients. Neurosurg Rev 24:8-13

68. Yoon HJ, Song YG, Park WI, Chol JP, Chanh KH, Kim JM (2004) Clinical manifestations and diagnosis of extrapulmonary tuberculosis. Yonsei Med J 45:453-461

69. Zimmerli W (2010) Vertebral osteomyelitis. New Eng J Med 362:1022-1029

70. Zink A, Chass CJ, Reisch U, Szeimies U, Nerlich AG (2001) Molecular analysis of skeletal tuberculosis in an ancient Egyptian population. J Med Microbiol 50:355-566

71. Zink AR, Grabner W, Nerlich AG (2005) Molecular identification of human tuberculosis in recent and historic bone tissue samples. The role of molecular techniques for the study of historic tuberculosis. Am J Phys Antropol 126:32-47

72. Weng $\mathrm{CH}$, Chi $\mathrm{CH}$, Shih $\mathrm{P}$, Ho CH, Po Lin, Chou $\mathrm{CH}$, Wang JH, Ho MW (2010) Spinal tuberculosis in non-HIV infected patients: a 10-year experience of a medical centre in central Taiwan. J Microbiol Immunol Infect 43:464-469 